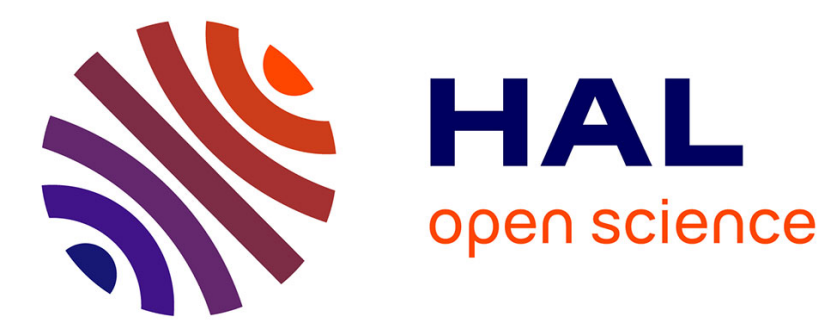

\title{
Single Linear Integral Action Control for Closed-loop Positioning of a Biomimetic Actuator with Artificial Muscles
}

\author{
B. Bonnaud, L. Escande, Bertrand Tondu, Philippe Souères
}

\section{- To cite this version:}

B. Bonnaud, L. Escande, Bertrand Tondu, Philippe Souères. Single Linear Integral Action Control for Closed-loop Positioning of a Biomimetic Actuator with Artificial Muscles. European Control Conference, Jul 2015, Linz, Austria. hal-02004969

\section{HAL Id: hal-02004969 \\ https://hal.science/hal-02004969}

Submitted on 3 Feb 2019

HAL is a multi-disciplinary open access archive for the deposit and dissemination of scientific research documents, whether they are published or not. The documents may come from teaching and research institutions in France or abroad, or from public or private research centers.
L'archive ouverte pluridisciplinaire HAL, est destinée au dépôt et à la diffusion de documents scientifiques de niveau recherche, publiés ou non, émanant des établissements d'enseignement et de recherche français ou étrangers, des laboratoires publics ou privés. 


\title{
Single Linear Integral Action Control for Closed-loop Positioning of a Biomimetic Actuator with Artificial Muscles
}

\author{
B. Bonnaud, L. Escande, B. Tondu and P.Soueres
}

\begin{abstract}
Artificial muscle is a new type of biomimetic actuator particularly promising for naturally compliant mechatronic and robotic systems. Due to their highly nonlinear character, their accurate closed-loop positioning control is a true challenge. We report the design and the control of a wire-cable driven artificial muscle actuator inspired by the elbow natural musculature. We propose an original linear control approach deriving benefit from natural stiffness and damping of any artificial muscle. We show how a single I-linear controller can be a practical simple way for an accurate and robust closed-loop positioning control. Experimental results have been performed by using hand-made pneumatic McKibben artificial muscles.
\end{abstract}

\section{INTRODUCTION}

Artificial muscle is a new type of actuator particularly promising for naturally compliant mechatronic and robotic systems. Whatever the considered technology, their soft character, based on the use of soft materials, generally makes them highly non-linear. Their accurate closed-loop positioning control is, consequently, a true challenge which has been answered differently over the two last decades: adaptive control [1], sliding mode control [2]-[4], fuzzy logic [5], neural network [6] or model-based approaches [7]-[8]. These sophisticated approaches have two major drawbacks: one the one hand, they often require a large number of parameters to tune or rules to specify; on the other hand, their excellent performances, for a given set of parameters or rules, are often limited to a relatively narrow range of motions. This is why some authors have also chosen classic PID control [9]-[10] with a part of purely intuitive tuning of the parameters. For example - and this was verified in the case of our actuator - the classic Ziegler-Nichols method can fail due to the highly non-linear character of the considered system. In a recent paper, we analyzed the relevance of a single linear I-controller for the closed-loop positioning of one single artificial muscle and we reported promising experimental results with a pneumatic McKibben muscle [11]. This is a similar approach we now want to apply to the case of a revolute actuator made of antagonist muscles. After introducing, in section 2, our original biomimetic experimental set-up, we develop in section 3 a simplified actuator model to which is applied a first stability analysis of the closed-loop controller; finally experimental results using desired step and sine wave signals, with and without load, are reported.

B.Bonnaud and L.Escande are $4^{\text {th }}$ year students at the Electrical Engineering Department at the Institut National de Sciences Appliquées (INSA), University of Toulouse, Campus de Rangueil, 31077 Toulouse; B.Tondu is with the INSA/Toulouse and LAAS/CNRS; P.Soueres is with the LAAS/CNRS, 7 avenue du colonel Roche, 31400, Toulouse, France (email: bertrand.tondu@insa-toulouse.fr). This paper was partially supported by the European project EUROC.

\section{ELBOW-LIKE EXPERIMENTAL SET-UP ACTUATED BY PNEUMATIC MCKIBBEN MUSCLES}

Flexion of human elbow is mainly realized by means of three muscles, as illustrated in Fig. 1 : the brachialis, whose origin is located at the lower half of the humerus, is inserted on the ulna; the brachio-radialis, whose origin is located at the supracondylar ridge of the humerus, is inserted on the radius; and the biceps brachii, which is the main flexor of the elbow, is inserted on the radial tuberosity; it arised not from the humerus but from the scapula; it is composed of a long and a short heads.

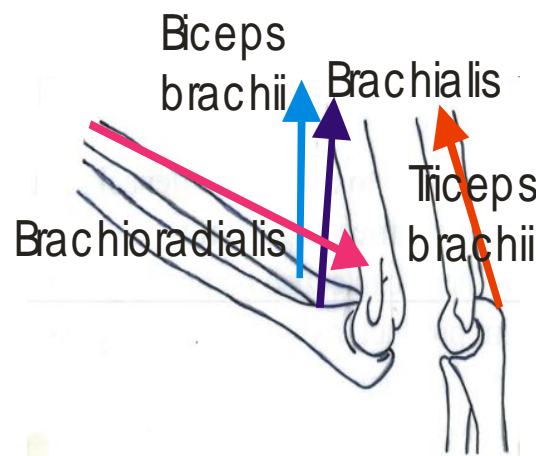

Figure 1. Schematic representation of the main elbow flexors and extensors (simplified and redrawn from [12]).

Some twenty years ago, the Biorobotics Laboratory of Washington [13] attempted to develop a highly biomimetic McKibben-type artificial muscle architecture mimicking such complex elbow musculature supplemented by that of the shoulder. However no control results were, at our knowledge, reported. Recently, biomimetic non-pulley musculoskeletal systems were designed [14] but actuated by classic linear actuators. Before attempting by ourselves to mimic the elbow natural musculature with four or five different muscles, we wanted to test the possibility to design a simplified antagonistic structure and to verify the possibility to control it in a simple and efficient way. By comparison with the classic pulley-chain approach, illustrated further in Fig. 3.a, the originality of our design approach consists to directly attach the muscles to the mobile link, which is a preliminary for considering a further multiple muscle joint structure. In our experimental set-up shown in Fig. 2, both biceps and triceps are attached to the forearm by means of a wire and hook system. The "point of insertion" of the muscles can be tuned by means of a sliding guide attachment system. But if changing biceps attachment point directly modifies the distance - noted further $d$ - from joint centre to biceps muscle force direction and so the resulting actuator torque, the distance - noted further $r$ - from joint centre to triceps muscle force direction is imposed to be constant by means of a circular guide. Biceps and triceps muscles are made of 
hand-made pneumatic McKibben muscles: only one biceps is considered with an active initial length of about $50 \mathrm{~cm}$, an initial section of about $2 \mathrm{~cm}^{2}$ and a maximum force of about $1000 \mathrm{~N}$; due to the mechanical structure of our actual experimental apparatus, two identical lateral triceps are considered with same initial section and maximum force than the biceps but an initial active length equal to about $33 \mathrm{~cm}$. By analogy with the elbow movement, joint actuator angle varies from a zero value, corresponding to alignment of arm and forearm, to a final full flexion angular position whose angle is less than $180^{\circ}$. In the initial position, biceps is fully elongated while triceps are fully contracted. An optical encoder is used for recording angular position and Labview software was used for programming the I-controller.

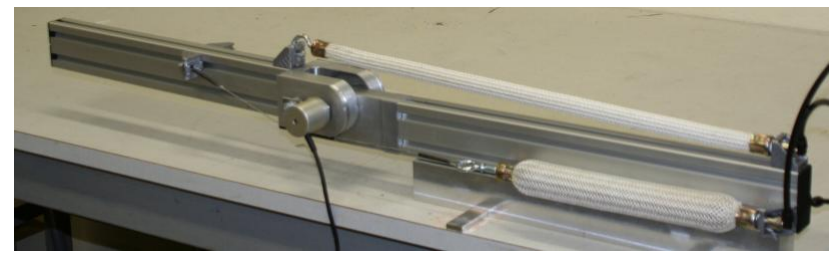

(a)

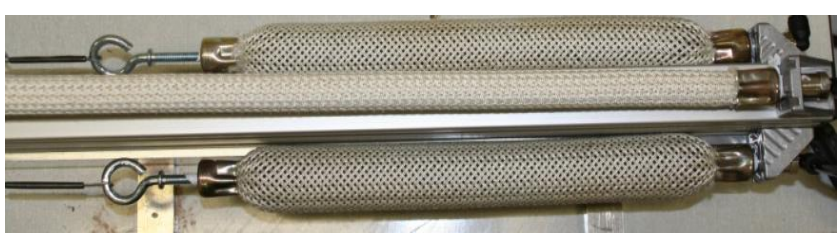

(b)

Figure 2. Photographs of the experimental set-up in zero-angle initial position, (a) Side view, (b) Top view showing the two lateral "triceps".

\section{MODELLING AND DIMENSIONING OF THE WIRE DRIVEN BIOMIMETIC ACTUATOR}

\section{A. Modelling}

In his seminal paper, N. Hogan considers that 'the entire group of muscles acting about the elbow are modeled by two opposing muscles acting at fixed moment arms about the joint axis' ([15], page 684), as illustrated in Fig. 3.a. This pulley-type approach is however questionable since it does not correspond to real physiology. This is why we propose to consider, inspired by other joint physiology kinematic studies, the alternative approach shown in Fig. 3.b: as in the case of Hogan's model, only two 'equivalent' muscles are considered, called biceps and triceps, but these two muscles are now directly attached to the links supposed to be reduced to line segments. It is worthy to note that this model is a rough approximation of our experimental set-up since it assumes two identical muscles while biceps and triceps muscles of the considered apparatus have different lengths and that our two identical triceps are equivalent to a single muscle with a twofold higher force. Furthermore, our real muscles are attached to links by means of mechanical parts which induce non-zero distance between the muscle tip and the link. We, consequently, considered this model as a first theoretical step for highlighting the fundamental properties of the actuator in open-loop and closed-loop without excessive formal developments. The model is organized as follows: on the one hand, biceps has its origin in point $\boldsymbol{A}$ on the fixed link (arm) and is inserted in $\boldsymbol{B}$ on the mobile link (forearm). The distance between $\boldsymbol{A}$ and the elbow joint centre $\boldsymbol{E}$ is noted $a$ and the one between $\boldsymbol{B}$ and $\boldsymbol{E}$ is noted $b$. On the other hand, it is supposed that the 'tendon' of the triceps is driven around the elbow joint center with a constant radius $r$. Let us note respectively $F_{b}$ and $F_{t}$ the forces produced by the biceps and the triceps, $\theta$ the elbow joint angle, and $T$ the torque generated by the two muscles. From a simple geometric analysis of Fig. 3.b, we deduce the following relationships:

$$
L(\theta)=\sqrt{a^{2}+b^{2}+2 a b \cos \theta}
$$

where $L(\theta)=A B$. Moreover, the distance $d$ from $\boldsymbol{E}$ to the line segment $[\boldsymbol{A} \boldsymbol{B}]$ is given by $d(\theta)=a b \sin \theta / L(\theta)$ and since $T=d(\theta) F_{b}-r F_{t}$, we deduce:

$$
T=\frac{a b \sin \theta}{\sqrt{a^{2}+b^{2}+2 a b \cos \theta}} F_{b}-r F_{t}
$$

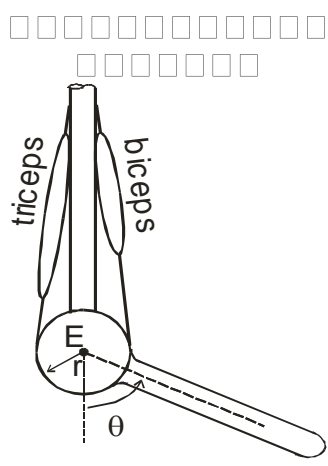

(a)

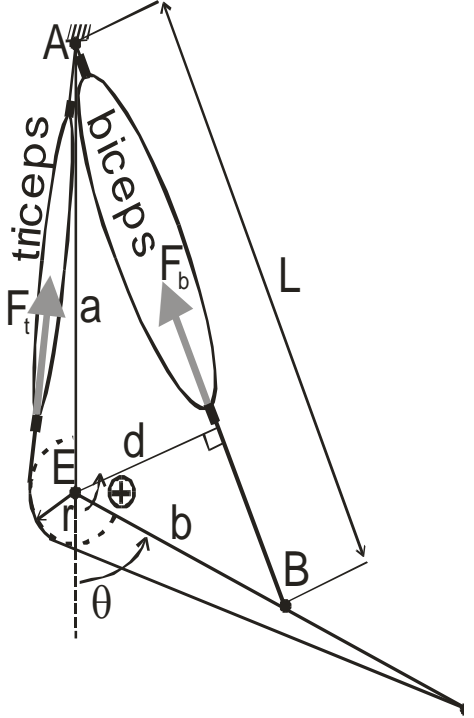

(b)
Figure 3. Proposed biomimetic model of the action of biceps and triceps around elbow joint (b) to be compared with the simplified pulley-chain model assuming fixed moment arms for biceps and triceps during joint movement (a) - adapted from [15], figure 1.c.

In order to determine a static model of the actuator, we will assume the following simplified static force model of the skeletal muscle $F(u, \varepsilon)$ :

$$
F(u, \varepsilon)=u F_{\max }\left(1-\frac{\varepsilon}{\varepsilon_{\max }}\right)
$$

where $u$ is a normalized control variable between 0 and 1 , generating a maximum force $F_{\max }$ for $u=1$ and $\varepsilon$ is the contraction ratio of the muscle defined as its length variation over its initial length, and varying between 0 and a maximum $\varepsilon_{\text {max }}$-value. Such a model captures the fundamental variable stiffness spring nature of the skeletal muscle without however being able to take into account the passive tension peculiar to the skeletal muscle. Because our closed-loop control, in the framework of this paper, is a SISO-control we will consider a symmetrical control variation between the agonist and antagonist muscles. In the initial state, the system is supposed to be at a $\theta$-angle equal to zero and because this angle can 
only vary inside the positive range $\left[0, \theta_{\max }\right]$, we impose, in the initial position, a control value for $u$ equal to 1 for biceps and to 0 for triceps. Let us consider a length variation for the contracted biceps defined by $\Delta L(\theta)=L(0)-L(\theta)=a+b-L(\theta)$. Since the radius $r$ is constant, the corresponding elongation of the triceps is equal to $r \theta$. Because the considered muscles are supposed to have the same initial length $l_{0}$, the following static force model results for biceps and triceps:

$$
\left\{\begin{array} { l } 
{ F _ { b } = u F _ { \operatorname { m a x } } ( 1 - \varepsilon _ { b } / \varepsilon _ { \operatorname { m a x } } ) } \\
{ F _ { t } = ( 1 - u ) F _ { \operatorname { m a x } } ( 1 - \varepsilon _ { t } / \varepsilon _ { \operatorname { m a x } } ) }
\end{array} \text { with } \left\{\begin{array}{l}
\varepsilon_{\mathrm{b}}=(a+b-L(\theta)) / l_{0} \\
\varepsilon_{\mathrm{t}}=\varepsilon_{\max }-r \theta / l_{0}
\end{array}\right.\right.
$$

By combining "(2)" and "(4)", we deduce the following expression for static torque, noted $T_{\text {static }}$ :

$$
\begin{aligned}
& T_{\text {static }}=f(\theta) u+g(\theta) \quad \text { with: } \\
& \left\{\begin{array}{l}
\left.f(\theta)=F_{\max }\left[d(\theta)(1-\Delta L(\theta)) / l_{0} \varepsilon_{\max }\right)+\left(r^{2} \theta\right) / l_{0} \varepsilon_{\max }\right] \\
g(\theta)=-F_{\max }\left(r^{2} \theta\right) /\left(l_{0} \varepsilon_{\max }\right)
\end{array}\right.
\end{aligned}
$$

We show in Fig. 4 the simulation of this static torque versus joint angle, for a constant control variable, and following data: $F_{\max }=1000 \mathrm{~N}, \varepsilon_{\max }=0.25, l_{0}=a=0.5 \mathrm{~m}, b=0.05 \mathrm{~m}$, $r=0.025 \mathrm{~m}$. The torque is generated until a maximum joint angle imposed by the two constraints: $\varepsilon_{b} \leq \varepsilon_{\max }$ and $\varepsilon_{t} \geq 0$. It is interesting to note the similarity of such curves with the defining static characteristic of the human elbow joint.

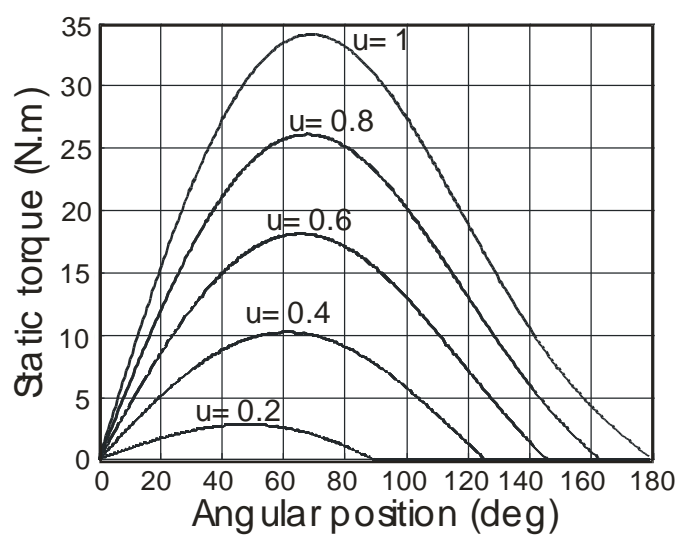

Figure 4. Static actuator characteristic at constant $u$-control value.

Positioning of such an actuator is stable in open-loop through the following relationship: $T_{\text {static }}=f(\theta) u+g(\theta)=0$, determining a unique non-zero equilibrium angular position. When joint is moved from this equilibrium position, a

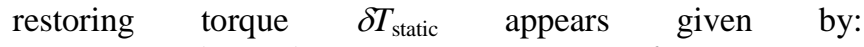
$\delta T_{\text {static }}=\left[g^{\prime}(\theta)-\left(f^{\prime}(\theta) g(\theta) / f(\theta)\right)\right] \delta \theta$ where $f^{\prime}(\theta)$ and $g^{\prime}(\theta)$ designate respectively the derivative functions of $f$ and $g$ with respect to $\theta$. It is easy to check that for $\delta \theta>0, \delta T_{\text {static }}<0$ and so the actuator stiffness, noted $S(\theta)$, depending on current angular position, can be defined as follows (see Fig. 6):

$$
S(\theta)=\left[f^{\prime}(\theta) g(\theta) / f(\theta)\right]-g^{\prime}(\theta)
$$

\section{B. Dimensioning}

We want now to understand how to specify the actuator dimensions in order to reach a given joint range $\left[0, \theta_{\max }\right]$. In the case of our actuator the maximum reachable angle is obtained for $\varepsilon_{b}=\varepsilon_{\max }$ - assuming that the biceps can fully contracted i.e. reaching $\varepsilon_{\max }$ while $\varepsilon_{t} \geq 0$ - and a control value equal to 1 . It is worthy to note that this analysis only depends on the biceps characteristics and, consequently, can be directly applied to our apparatus. From $\varepsilon_{\mathrm{b}}$ definition, we get: $\Delta L\left(\theta_{\max }\right)=l_{0} \varepsilon_{\max }$, from which is derived the following relationship giving the ratio $(b / a)$ required for reaching the $\theta_{\max }$ angle, for a given $\left(l_{0} / a\right)$-ratio and a given $\varepsilon_{\max }$ with, however, the additional constraint $l_{0} \leq(a+b)$ :

$$
\frac{b}{a}=\frac{\left(l_{0} / a\right) \varepsilon_{\max }\left[1-0.5\left(l_{0} / a\right) \varepsilon_{\max }\right]}{1-\cos \left(\theta_{\max }\right)-\left(l_{0} / a\right) \varepsilon_{\max }}
$$

For $\varepsilon_{\max }=0.25$ and $\theta_{\max }=145^{\circ}$, we show in Fig. 5 the corresponding $(b / a)$-ratio versus $\left(l_{0} / a\right)$-ratio. Because any choice of $a$ and $b$ parameters directly influences the maximum torque by the intermediate of distance $d$, it is necessary to put in correspondence the $(b / a)$-ratio with another relationship relating the maximum torque to the $\left(l_{0} / a\right)$-ratio. On Fig. 4 , it is clear that torque is maximum for $u=1$ and an intermediate angle $\theta_{\text {ext }}$ numerically determined. From: $T_{\max }=d\left(\theta_{\text {ext }}\right) F_{b}\left(u=1, \theta=\theta_{\text {ext }}\right)$, we derive:

$$
\frac{T_{\max }}{a F_{\max }}=\left[\frac{(b / a) \sin \left(\theta_{\text {ext }}\right)}{\left(L\left(\theta_{\text {ext }}\right) / a\right)}\right]\left[1-\frac{1+(b / a)-\left(L\left(\theta_{\text {ext }}\right) / a\right)}{\left(l_{0} / a\right) \varepsilon_{\max }}\right]
$$

with: $L\left(\theta_{\text {ext }}\right) / a=\sqrt{1+(b / a)^{2}+2(b / a) \cos \left(\theta_{\text {ext }}\right)}$. From Fig. 5 it appears that, for a given $a$-length, the best choice consists in choosing $l_{0}=(a+b)$ and a $(b / a)$-ratio equal to about 0.13 , for $\varepsilon_{\max }=0.25$ and $\theta_{\max }=145^{\circ}$. In the special case of the McKibben muscle technology, it is however not possible to impose $l_{0}=(a+b)$ due to the muscle tips. As can be seen in Fig. 2.a, we tried to minimize the lost non-active length for the biceps between points $\boldsymbol{A}$ and $\boldsymbol{B}$ with as a result a $\left(l_{0} / a\right)$ close to 1 and a $(b / a)$ equal to about 0.14 . Finally we checked a maximum reachable angle of about $120^{\circ}$ and, after several trials, no other $l_{0}, a$ and $b$ combination was able to give more. According to us, this discrepancy between theory and experiment is mainly due to the fact that no normal offsets have been taken into account in our simplified model. The obtained range was however large enough to test the performances of the proposed controller.

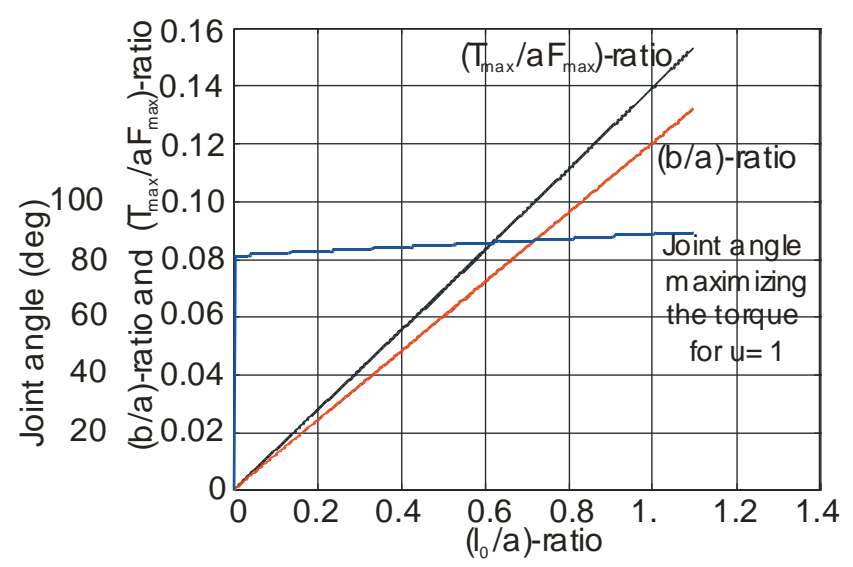

Figure 5. Dimensioning of the geometric actuator parameters (see text). 


\section{Closed-LOOP POSITIONING OF THE ACTUATOR BASED ON ARTIFICIAL MUSCLE STIFFNESS}

The $u$-control variable of our I-controller will be specified as follows:

$$
u(t)=k_{I} \int_{0}^{t}\left(\theta_{d}(\tau)-\theta(\tau)\right) d \tau
$$

where $k_{I}$ is a constant gain. We first propose to develop a stability analysis of the controller applied to the previous considered actuator model, before showing how in practice it works.

\section{A. Stability Analysis}

The following dynamic model of the actuator is considered, where $T_{\text {static }}$ is the actuator static torque expressed in "(5)" and $f_{v}$ a viscous coefficient dedicated to approximate complex kinetic friction inside the artificial muscle by a linear viscous damping:

$$
T_{d y n}=T_{\text {static }}-f_{v} \&
$$

Moreover, it is assumed that the actuator is moving a given inertia $I$ i.e. $T_{d y n}=I \&$ and that the moving parts of the muscles have negligible inertias by comparison to the one of the moving link.

Let us put the I-control law into "(5)" with a constant desired position $\theta_{\mathrm{d}}$, and let us derive from "(10)" the resulting dynamic expression with respect to time. We get:

$$
\left[u(t) f^{\prime}(\theta)+g^{\prime}(\theta)\right] \& k_{I}\left(\theta_{d}-\theta\right) f(\theta)=f_{v} \& I \&
$$

Equilibrium positions of the system correspond to angular positions noted $\theta_{\text {equ }}$ in which $\&_{e q u}^{\&}=\&_{e q u}=\&_{e q u}=0$. We should have:

$$
k_{I}\left(\theta_{d}-\theta_{e q u}\right) f\left(\theta_{e q u}\right)=0
$$

From $\Delta L\left(\theta_{\max }\right)=l_{0} \varepsilon_{\max }$, we derive: $f\left(\theta_{\text {equ }}\right)=$ $F_{\text {max }}\left[d\left(1-\Delta L\left(\theta_{\text {equ }}\right) / \Delta L\left(\theta_{\text {max }}\right)\right)+r^{2} \theta_{\text {equ }} / \Delta L\left(\theta_{\text {max }}\right)\right], \quad$ and since $\Delta L\left(\theta_{\text {equ }}\right) \leq \Delta L\left(\theta_{\max }\right)$, we deduce $f\left(\theta_{\text {equ }}\right)>0$ and then:

$$
\theta_{d}=\theta_{e q u}
$$

Let us now determine under which conditions the system is stable in the desired position. Due to its non-linear character, we develop a classic differential analysis around the equilibrium position. Let us put the following state variables: $x_{1}=\&, x_{2}=\&, x_{3}=\theta-\theta_{d}$ and the equilibrium state vector with components $x_{1 d}=x_{2 d}=x_{3 d}=0$ from which we move with respective small deviations $\varepsilon_{1}, \varepsilon_{2}, \varepsilon_{3}$ i.e. $x_{i}=x_{i d}+\varepsilon_{i}$, for $i=1,3$. We can assume that deviation from equilibrium has a short duration by comparison to the time required to put the joint at the desired position and consequently we will assume that, during deviation, the control $u$ is constant and equal to the $u_{\mathrm{d}}$-control value for maintaining the joint at the desired position. From " $(5)$ " we derive: $u_{d}=-g\left(\theta_{d}\right) / f\left(\theta_{d}\right)$. Вy using this last relationship and stiffness definition of "(6)", we can re-rewrite "(11)" as follows:

$$
-f_{v} x_{1}-S\left(\theta_{d}\right) x_{2}-k_{I} f\left(\theta_{d}\right) x_{3}=I \&
$$

and so the following relationship results : $[\&, \&]^{T}=\boldsymbol{J}\left[x_{1}, x_{2}, x_{3}\right]^{T}$ where $\boldsymbol{J}$ is equal to:

$$
\boldsymbol{J}=\left[\begin{array}{ccc}
-f_{v} / I & -S\left(\theta_{d}\right) / I & -k_{I} f\left(\theta_{d}\right) / I \\
1 & 0 & 0 \\
0 & 1 & 0
\end{array}\right]
$$

whose characteristic polynomial is given by:

$$
I \lambda^{3}+f_{v} \lambda^{2}+S\left(\theta_{d}\right) \lambda+k_{I} f\left(\theta_{d}\right)=0
$$

By a simple application of Ruth's criteria applied to a third order linear model, we deduce the stability condition:

$$
k_{I}\left(\theta_{d}\right)<k_{I \_l i m}\left(\theta_{\mathrm{d}}\right) \text { with } k_{I \_l i m}\left(\theta_{\mathrm{d}}\right)=f_{v} S\left(\theta_{d}\right) /\left[I f\left(\theta_{d}\right)\right]
$$

We show in Fig. 6 how $k_{I \_l i m}$ varies with joint angle for data already considered in Fig. 4 with also: $b=0.14 a, f_{v}=100 \mathrm{~N} / \mathrm{rd}$, $l_{G}=0.25 \mathrm{~m}$ and $I=0.045 \mathrm{~kg} \cdot \mathrm{m}^{2}$. Due to our model without muscle attachment orthogonal distance, the biceps generates no force at a zero joint angle making the system instable at $\theta_{d}=0$. We so considered a joint angle greater than $20^{\circ}$. It clearly appears that a $k_{I-l i m}$ value can be deduced for being adapted to the full considered joint range. Moreover, if we know a priori that the inertia $I$ can vary under a given limit $I_{\max }$, the $k_{I \text { lim }}$ can be tuned from the datum of $I_{\max }$.

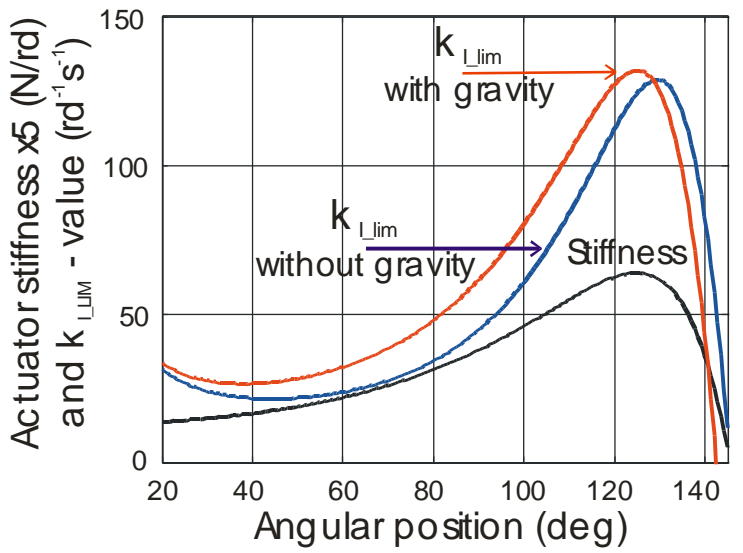

Figure 6. Actuator stiffness and limit-values for I-action gain.

Let us now consider the gravity effect under the form the following torque: " $-M g l_{G} \sin (\theta)$ " where $M g$ is the mobile link load and $l_{G}$ the distance from the joint centre to the gravity link centre. Equation "(11)" is now modified into:

$$
\begin{array}{r}
{\left[u(t) f^{\prime}(\theta)+g^{\prime}(\theta)-M g L_{G} \operatorname{co}(\theta)\right] \& k_{I}\left(\theta_{d}-\theta\right) f(\theta)} \\
=f_{v}
\end{array}
$$

By applying the same reasoning as in the case without gravity, the new limit-value for $k_{I}$ results:

$$
k_{I \_\lim }=\frac{f_{v}}{I f\left(\theta_{d}\right)}\left[S\left(\theta_{d}\right)-M g L_{G}\left(\frac{\sin \left(\theta_{d}\right) f^{\prime}\left(\theta_{d}\right)-\cos \left(\theta_{d}\right) f\left(\theta_{d}\right)}{f\left(\theta_{d}\right)}\right)\right]
$$

As shown in Fig. 6, the two curves exhibiting $k_{I_{-} \text {lim }}$ with and without gravity are very close. 
Our I-controller stability analysis is not, rigorously spoken, a proof of stability, that we plan to do in a future work by looking for an adequate Lyapunov's function, but it justifies, according to us, the practical possibility for tuning the Iaction gain for any naturally damped given artificial muscle.

\section{B. Experimental results}

We report, first, closed-loop step responses with gravity effect but no additional load. The same $k_{I}=0.005 \mathrm{bar} / \mathrm{rd}$.s was used for the five reported desired angles: $20^{\circ}, 40^{\circ}, 60^{\circ}, 80^{\circ}, 100^{\circ}$, with the look for a compromise between short response time and limited maximum overshooting. As shown in Fig. 7.a, a response time of about $1 \mathrm{~s}$ is obtained; maximum overshoot is limited to $5 \%$ expect for the $20^{\circ}$-step in accordance with the fact theoretically highlighted that the system is closer to instability at weak desired angles. Dry friction peculiar to McKibben muscle technology induces specific non-linear phenomena [16] but thanks to the integral action, as can be shown in Fig. 7.b, steady state error belongs to the $\left[-0.3^{\circ},+0.3^{\circ}\right]$-range.

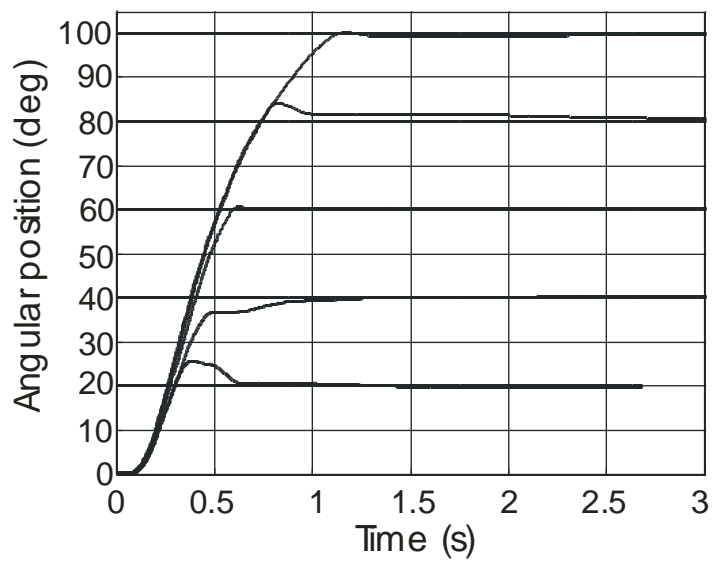

(a)

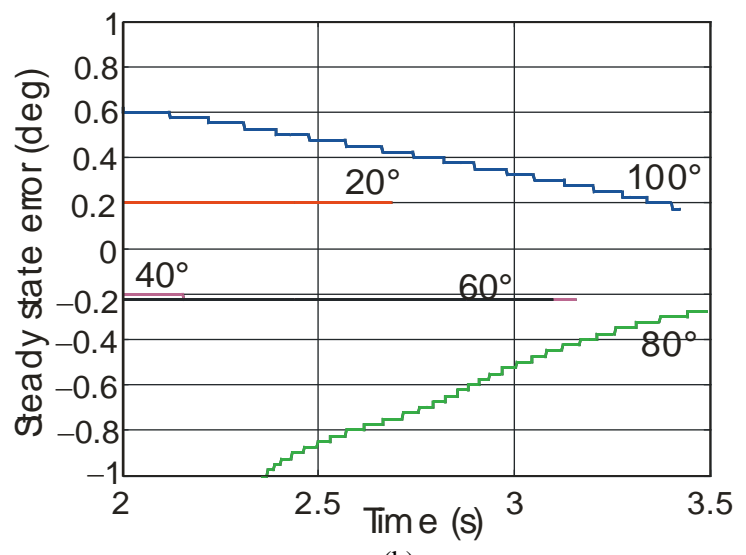

(b)

Figure 7. Step responses for various desired joint angles (a) and corresponding steady state errors (b).

We now consider the particular case of a desired $90^{\circ}$ position which can be considered as a critical case since torque due to gravity changes sign at this position, and we put additional loads at the tip of the mobile link, as shown in Fig. 8.a while keeping the value of the $k_{I}$-gain equal to the previous one: 4 loads equal to $0.2,0.4,0.6$ and $0.8 \mathrm{~kg}$ were considered. Loads respectively represent a change of inertia equal to about $100 \%, 200 \%, 300 \%$ and $400 \%$ the initial mobile link inertia and an additional torque due to gravity respectively equal, in absolute value, to $10 \%, 20 \%, 30 \%$ and $40 \%$ the initial mobile link gravity torque. As it can be seen in Fig. 8.b very close performances are obtained with 0.2 and $0.4 \mathrm{~kg}$ loads by comparison with response without load; for upper loads the system oscillates but remains stable with, in all cases, a steady state error similar to this without load.

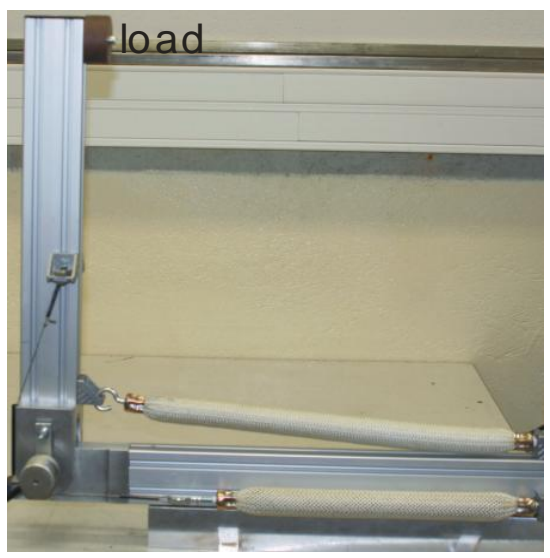

(a)

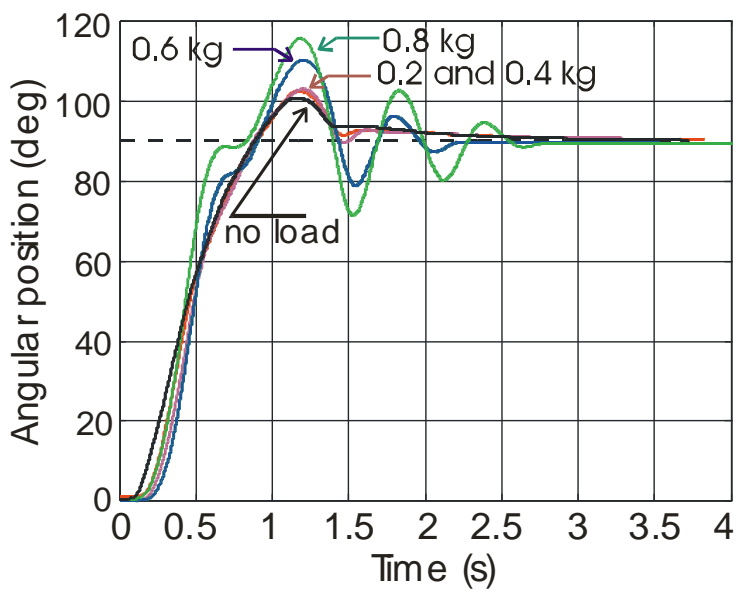

(b)

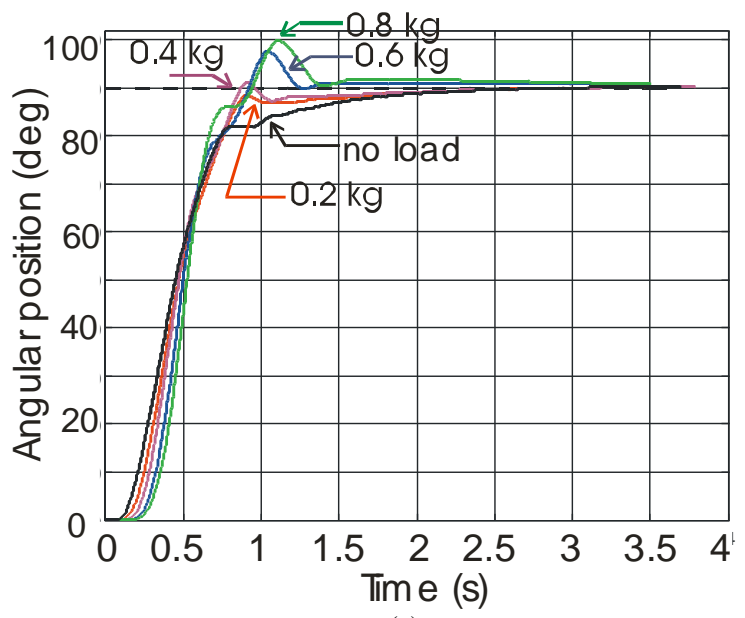

(c)

Figure 8. Load robustness analysis of the actuator for a $90^{\circ}$-step response, (a) Photograph of the experimental set-up with a load of $0,4 \mathrm{~kg}$ at the tip of the mobile link, (b) Step responses with the four considered loads,

(c) Effect of a change of $k_{l}$-gain from 0.005 to $0.004 \mathrm{bar} / \mathrm{rd} . \mathrm{s}$. 
We show in Fig. 8.c the effect of a small change for the $k_{I^{-}}$ gain: overshoot is now limited to almost $10 \%$ in all load cases with a similar response time of about $2 \mathrm{~s}$, but we noticed elsewhere that this lower gain-value can generate higher steady state errors for larger steps with no load due to dry friction inside McKibben muscles.

Finally, the actuator is tested in response to two sine waves: between $40^{\circ}$ and $80^{\circ}$, and between $20^{\circ}$ and $100^{\circ}$. In both cases a time period of 5 seconds was considered and a same $k_{I}$-gain of $0.025 \mathrm{bar} / \mathrm{rd}$.s. As shown in Fig. 9, maximum tracking error occurs at the change of direction due to muscle dry friction but also due to friction at attaching hook (an improved version of the set-up would try to prevent this mechanical difficulty). If we now manually stop the mobile link, for example at its lower angular position during about 2 seconds, the mobile link relatively quickly comes back to the desired trajectory without excessive oscillations.

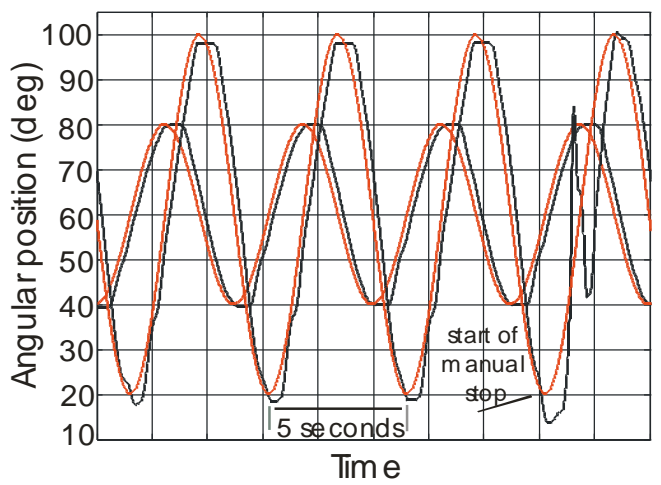

Figure 9. Sine wave tracking and effect of a manual perturbation - desired sine wave trajectories are shown in red.

\section{CONCLUSION AND FUTURE WORK}

We reported experimental results for a non-linear actuator with artificial muscles controlled by a simple I-linear action. We justified this approach by a preliminary stability analysis based on the fact that any artificial muscle actuator is characterized by its own stiffness and its own damping. In practice, due to the difficulty to accurately model any artificial muscle actuator, the tuning of the $k_{I}$-gain is made empirically but, because there is only one gain to tune, we think that this approach is meaningful. By comparison with a classic PID, it is possible that, in some particular desired signal case, a PID would locally give a better result but the look for a relevant choice in a large motion range is much more difficult to determine than with our single I-action. We checked the relevance of this approach in the case of the pneumatic McKibben artificial muscle known to be particularly non-linear due to complex friction phenomena during contraction. Further work will try to rigorously establish the stability proof by exhibiting a Lyapunov function adapted to a general model of artificial muscle as to our specific apparatus. We also have the ambition to enlarge our SISO control approach to a combined position-stiffness MIMO control approach deriving benefit of the simplicity of our closed-loop position control law. These efforts will be made on some improved mechanical version of our actual biceps-triceps system, and on a new one with multiple muscles mimicking the naturally redundant musculature. In particular, in the case of our elbow-like actuator, it could be wondered if an additional anconeus-like artificial muscle could increase the joint range and/or the load robustness.

Beyond the hopped development of accurate and robust naturally compliant mechatronic or robotic actuation systems, the possibility to closed-loop control, in a stable way, biomimetic artificial muscle systems poses the question about the possible role of equivalent position error integrators inside the natural locomotor system.

\section{REFERENCES}

[1] D.G. Caldwell, G.A. Medrano-Cerda, and M. Goodwin, "Control of pneumatic muscle actuators," IEEE Control Systems Magazine, vol. 15, no. 1 , pp. 40-48, 1995.

[2] B. Tondu, and P. Lopez, "Modeling and control of McKibben artificial muscle robot actuators", IEEE Control Systems Magazine, vol. 20, no. 2, pp. 15-38, 2000.

[3] D. Cai D, and Y. Dai, "A sliding mode controller for manipulator driven by artificial muscle actuator", Electronics and Communications in Japan, vol. 86, no. 11, pp. 290-297, 2003.

[4] K. Braikia, M. Chettouh, B. Tondu, P. Acco, and M. Hamerlain, "Improved control strategy of 2-sliding controls applied to a flexible robot arm", Advanced Robotics, vol. 25, pp. 1515-1538, 2011.

[5] H.P.H. Anh, and K.K. Ahn, "Hybrid control of a pneumatic artificial muscle robot arm using an inverse NARX fuzzy model," Eng. Appli. of Artificial Intelligence, vol. 24, no. 4, pp. 697-776, 2011.

[6] S. Tian, G. Ding, D. Yan, L. Lin, and M. Shi, "Nonlinear controlling of artificial muscle system with neural networks," in Proc. of the 2004 IEEE Int. Conf. on Robotics and Biomimetics, Shenyang, China, pp. 56-59, 2004.

[7] T.V. Minh, T. Tjahjowidodo, H. Ramon, and H. Van Brussel, "Control of pneumatic artificial muscle (PAM) with model-based hysteresis compensation," in Proc. of the IEEE/Adv. Int. Mechatronics Conf., Singapore, pp 1082-1087, 2009.

[8] X. Shen, "Nonlinear model-based control of pneumatic artificial muscle servo systems," Control Engineering Practice, vol. 18, no. 3, pp. 311-317, 2010.

[9] R.C. Richardson, M.C. Levesley, M.D. Brown, J.A. Hawkes, K. Watterson, and P.G. Walker, "Control of ionic polymer metal composites", IEEE/ASME Transactions on Mechatronics, vol. 8, no. 2, pp. 245-253, 2003.

[10] G. Gilardi, E. Haslam, V. Bundhoo, and E.J. Park, "A shape memory alloy based tendon-driven actuation sytem for biomimetic artificial fingers, part II: modelling and control," Robotica, vol. 28, no. 5, pp. 675-687, 2010.

[11] B. Tondu, "Robust and accurate closed-loop control of McKibben artificial muscle contraction with a linear single integral action," Actuators, , vol. 3, special Issue on Soft Actuators, pp. 142-161, 2014.

[12] Kahle W., Leonhardt H. and Platzer W., Anatomy, Vol. 1: Locomotor System, Georg Thieme Verlag, Stuttgart, Germany, 1975 (in German).

[13] B. Hannaford, J.M. Winters, C.-P. Chou, and P. Marbot, "The anthroform biorobotic arm: A system for the study of spinal circuits," Ann. Biomed. Eng., vol. 23, pp. 399-408, 1995.

[14] H. Kino, S. Kibuchi, Y. Matsutani, K. Tahara, T. Nishiyama, "Numerical analysis of feedforward position control for non-pulley musculoskeletal system: A case study of muscular arrangements of a two-link planar system with six muscles," Advanced Robotics, vol. 27, no. 16, pp. 1235-1248, 2013.

[15] N. Hogan, "Adaptive control of mechanical impedance by coactivation of antagonist muscles," IEEE Trans. Automatic Control, vol. AC-29, no. 8, pp. 681-689, 1984.

[16] B. Tondu, "Modelling of the McKibben artificial muscle: A review," Journal of Intelligent Materials Systems and Structures, vol. 23, no. 3, special issue on Fluidic Actuators, pp. 225-253, 2012. 